Article

\title{
Omnidirectional WPT and Data Communication for Electric Air Vehicles: Feasibility Study
}

\author{
Safa Zouaoui ${ }^{1,2}$, Wael Dghais ${ }^{2,3}$, Rui Melicio ${ }^{4,5, *}$ and Hamdi Belgacem ${ }^{1,2}$ \\ 1 Faculte des Sciences de Monastir, Université de Monastir, 5000 Monastir, Tunisia; \\ safa_zouaoui@yahoo.com (S.Z.); belgacem.hamdi@gmail.com (H.B.) \\ 2 Laboratoire d'Electroniques et Microelectroniques, University of Monastir, 5000 Monastir, Tunisia; \\ waeldghais@ua.pt \\ 3 Institut Supérieur des Sciences Appliquées et de Technologie de Sousse, Universite de Sousse, \\ 4003 Sousse, Tunisia \\ 4 IDMEC, Instituto Superior Técnico, Universidade de Lisboa, 1000 Lisboa, Portugal \\ 5 ICT, Escola de Ciências e Tecnologia, Universidade de Évora, 7000 Évora, Portugal \\ * Correspondence: ruimelicio@gmail.com; Tel.: +35-126-674-5372
}

Received: 9 October 2020; Accepted: 3 December 2020; Published: 8 December 2020

\begin{abstract}
This paper investigates the feasibility of using the three-dimensional omnidirectional inductive channel for power transfer and as a power line communication (PLC) for ground-based vehicle, electric air vehicle, or space applications. The simulation results were performed by the advanced design system software using lumped equivalent circuit model. The power transfer efficiency was determined based on multiport scattering (S)-parameters numerical simulation results while the theoretical channel capacity was calculated based on Matlab software as a function of the coupling coefficient considering an additive white Gaussian noise. Furthermore, the magnetic field distribution was evaluated as function of the misalignment angle $\theta$ between the receiver and the three orthogonal transmitters coils.
\end{abstract}

Keywords: 3D inductive channel; wireless power transfer; data communication; power line communication; energy efficiency; misalignment angle; coupling coefficients; finite elements; resonance data rate simulation; advanced design system (ADS) simulation; channel bandwidth; channel capacity; ground-based vehicle; electric air vehicle; space applications

\section{Introduction}

The significant growth of renewable energy generators [1,2] and the smart grid development have shaped the need for perpetual information exchange between the different constituents of a grid such as consumer electronic medical devices (CEMDs) [3-5]. Moreover, the continuous growth of battery assisted low-power handheld devices (e.g., smartphone or tablet, CEMDs) and the high-power battery assisted applications boosted mobility. In fact, user equipment (UEs) are frequently charging their mobile devices and with the deployment of the internet of things (IoT), the problems of ensuring charged batteries for portable devices will become more challenging [6]. In comparison to the ground-based vehicle, electric air vehicles, such as unmanned aerial vehicle (UAVs), drones, and all-electric aircraft (AEA), have attracted many researchers over the past decade $[7,8]$. UAVs are more practical to be utilized for applications such as package delivery and environmental monitoring [9]. On the other hand, the AEA has the advantage of low engine noise and emits much lower carbon dioxide if compared to traditional turbofan engines [8]. Until now, EVs are not so appealing to consumers due to their insufficient energy density, limited lifetime and high cost. For example, the commercialized lithium-ion batteries which are acknowledged as the most competitive solution used in EVs, still suffer 
from the lower energy density value; about $90-100 \mathrm{Wh} / \mathrm{kg}$ compared to gasoline which has about $12,000 \mathrm{Wh} / \mathrm{kg}$ [7]. In addition to that, the long charging time lasts from half an hour to serval hours for a single charge, according to the power level of the attached charger; a fact that can represent a danger with the risk of an electric shock in a specific environment $[7,8]$. Despite the widespread usage of UAVs and drones, usage in various civilian and commercial applications, their limited flight time, due to battery power limitation, restricts their space mobility and slows down their adoption and integration into 5G ecosystem $[7,8,10,11]$. Moreover, the current state-of-the-art of batteries technology still suffers from lower specific energy than fossil fuel. However, longer flight duration without recharging can be achieved only with a much larger battery capacity which unfortunately increases the overall payload weight and the total flight duration decreases. For these reasons, wireless power transfer (WPT) provides a potential solution for increasing the flying mission UAVs and assisting the 5G connectivity and performance, while integrating the smart grid network. Furthermore, WPT technology has been extensively adapted for advantages of convenience and safety. Since the 1960s, the WPT technique has been used in biomedical fields, which made great progress for human health care. The integration of WPT and data communication in medical devices increased the patients' mobility and facilitated the management of these devices because they can be charged while continuously monitoring the patient's health conditions by telemetering crucial data as well as ensuring high quality preventive and E-health life. For example, wireless charging scenario enables multiple electric air vehicle charging (AEA, UAV), which could increase the battery autonomy of the fleet of UAVs, whenever a critical situation arises that may require high data rate transmission or in search and rescue agency and assist civil security. Consequently, wireless charging technology enables higher autonomy and mobility in such scenarios with less interruption, by avoiding the need for going to charge and coming back to the mission. This is considered to be useful in various corporate activities, along with humanitarian and preventative industrial applications. In fact, achieving maximum power transfer efficiency between three-dimensional omnidirectional transmitting and receiving coils has proven challenging in maintaining high data rate.

WPT systems utilize coils to generate electromagnetic fields, which transfer power from the power base station into the coil embedded in UAVs. At high frequency, electromagnetic interferences (EMI) become an important issue where the high-intensity EMF can have a harmful impact not only on the surrounding equipment and the exchange of the data between the transceivers but also on the human safety. In fact, to figure out the range of interference generated by WPT equipment, serval studies are proposed [12-15]. Firstly, a study related to the electromagnetic emissions from WPT effects are discussed in $[12,13]$. However, it is crucial to assure that the EMI generated by the wireless power transfer system will not influence or disrupt the communication between the transmitter and the receiver [14,15]. Additionally, better optimization of the power and data links can be achieved by separating the frequency band of the data and power signal; the power signal carries no information and the power transfer efficiency is maximized for a narrow-band (e.g., high $Q$ ) link that usually operates at low frequencies. On the other hand, data signal is realized at much higher frequencies, particularly in the GHz order [14,15]. The experimental results of measurement and the analysis of the electromagnetic fields generated by WPT system as well as their possible interaction on data transmission channel are reported in $[13,14]$. They confirmed that there is no substantial impact from WPT system operating at $20 \mathrm{kHz}$ frequency on data transfer channel operating at $2.4 \mathrm{GHz}$ frequency. Moreover, with the integration of WPT technology in the high power applications such as EV charging, $\mathrm{UAV}$, drones, and AEA, the need of high transferred power increased. However, several studies focused on how to improve the system's performances [16-19]. Maximum transmission efficiency for WPT systems are reached under resonance condition, which is very sensitive to the load condition and the distances between the transceivers. However, to compensate the misalignments and distance variation between the Tx and Rx coils, authors proposed an efficient method of frequency tuning in [16] allowing the switching of the operating frequency of the prototype to the desired resonant frequency based on the software application. While in $[19,20]$, two magnetic core reactors (MCRs) are 
included at the transmitter and receiver sides to ensure the frequency control and to tune both ends at the same frequency, enabling the increase of the systems' performances by ensuring the impedance matching [16-18]. Furthermore, to advance the design of electric vehicles battery chargers using WPT technique, two different geometric configurations are compared in [21,22] in which the electrical three-phase system transmitter can be connected electrically and geometrically in a star or a delta configuration. In this paper, we focus on the analysis and design considerations of the induction-based wireless power supply and data communication through three-dimensional wireless channel inductive coupling for electric air vehicles such as UAV drones and AEA applications. The foremost contribution of this paper is to explore the feasibility study of using a three-dimensional wireless inductive channel for ensuring efficient WPT and Power Line Communication (PLC) by means of simulation and comparing power and data channel metrics. The proposed 3D induction wireless channel design investigates the effective integration of the data and power signals to share the same channel. The rest of this paper is organized as follows. Section 2 presents the concept of the omnidirectional WPT and the importance of data communication. Section 3 describes the current omnidirectional WPT porotypes. Section 4 presents the simulation results of the multiport S-parameters simulation to evaluate the performance of the power and data links. Finally, the conclusion is addressed in Section 5.

\section{Omnidirectional WPT and Data Communication}

The majority of WPT applications involve directional power flow and the concept of a coupled WPT-PLC system was recently demonstrated and analyzed [23-27]. In [23,25] a feasibility study of the whole system was performed, and the design guidelines were presented. Considering both data and power transmission requirements, the experimental setup was implemented and measured achieving theoretical capacities from tens of kbit/s to few Mbit/s; see for instance [24,28]. In this case, the receiving coil could be placed on parallel in fact, users cannot use their devices during charging and this is relatively flexible in terms of positioning. Meanwhile, the misalignment and displacement in a wireless inductive link can reduce the transferred power and efficiency and restrict the amount of power transfer. Toward overcoming this limitation, serval approaches have been proposed; first, multiple receiver coils structures are proposed to ensure that the coil's receiver can get enough power when there is some misalignment $[29,30]$. In fact, the multiple receiver coils structure approach is not preferred due its volumetric receiver coils structure and because it is not suitable for omnidirectional WPT systems. However, 3-D omnidirectional WPT can be treated as a prominent solution providing more freedom for the position of the receivers [31-34]. Firstly, omnidirectional WPT for 2-D systems was proposed in [34,35], in which the authors controlled the directivity of the maximum magnetic fields and efficiency by using the amplitude modulation. However, if the receiver's plane coil and the 2-D transmitting plane are parallel to each other, the receiver will be powerless. As a solution, 3-D omnidirectional was proposed in [33,36-39], and the mathematical analyses were considered in order to enhance the power delivery performances, besides, an omnidirectional magnetically coupled resonant WPT system and data communication is presented in [36] for preclinical studies. The analysis carried out in this manuscript is an extension of the work presented in a previous contribution [26].

\subsection{Wireless Charging Concept with Omnidirectional Magnetic Field}

The equivalent circuit modeling the 3D WPT system considered for the simulation analysis [36] is shown in Figure 1.

The three orthogonal TX coils are connected with the common AC power supply from class E power amplifier. The secondary coil, placed on-board the drone, must be very light. To this aim, a new WPT charging system was designed, focusing on the minimization of the weight, size, and magnetic field emission, while maintaining good electrical performances in power transfer. The coupling among the TX coils is neglected due to the orthogonal symmetry. $\left(M_{12}=M_{23}=M_{13}=0\right)$. The mutual inductances between the TX coils and Rx coil are represented as $M_{1 R}, M_{2 R}$, and $M_{3 R}$ where $M_{i R}=k_{i R} \sqrt{L_{i} L_{R}}, i \in\{1,2,3\}$. The omnidirectional power transfer method is presented to 
minimize the misalignment effect and to improve the uniform power flow to the Rx. Therefore, the simple three-orthogonal-coils structure is a better alternative for the omnidirectional transmitter while reducing the interferences between the three transmitters.

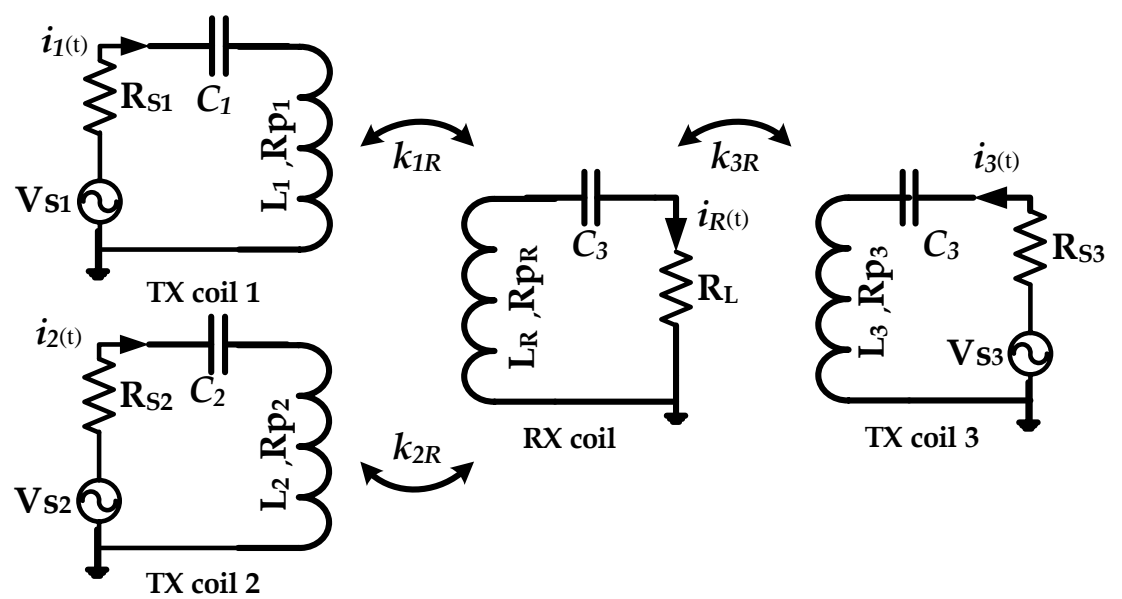

Figure 1. Lumped circuit model of omnidirectional wireless power transfer system [36].

Returning to the simplified model, where Kirchhoff's voltage law (KVL) can be utilized to determine the currents in every resonant circuit, where the first coil, the second coil, and the third coil of the transmitter are orthogonal to each other $j \omega M_{12}=j \omega M_{21}=j \omega M_{13}=j \omega M_{31}=j \omega M_{23}=j \omega M_{32}=0$, could be written as [37]:

$$
\left[\begin{array}{c}
V_{s 1} \\
V_{s 2} \\
V_{s 3} \\
0
\end{array}\right]\left[\begin{array}{cccc}
\left(R_{s 1}+R_{p 1}+j X_{1}\right) & 0 & 0 & j \omega M_{1 R} \\
0 & \left(R_{s 2}+R_{p 2}+j X_{2}\right) & 0 & j \omega M_{2 R} \\
0 & 0 & \left(R_{s 3}+R_{p 3}+j X_{3}\right) & j \omega M_{3 R} \\
j \omega M_{1 R} & j \omega M_{3 R} & j \omega M_{2 R} & \left(R_{L}+R_{p R}+j X_{R}\right)
\end{array}\right]\left[\begin{array}{c}
I_{1} \\
I_{2} \\
I_{3} \\
I_{R}
\end{array}\right]
$$

where

$$
\begin{aligned}
& \mathrm{X}_{1}=\omega L_{1}-\frac{1}{j \omega C_{1}} \\
& \mathrm{X}_{2}=\omega L_{2}-\frac{1}{j \omega C_{2}} \\
& \mathrm{X}_{3}=\omega L_{3}-\frac{1}{j \omega C_{3}} \\
& \mathrm{X}_{\mathrm{R}}=\omega L_{\mathrm{R}}-\frac{1}{j \omega C_{R}}
\end{aligned}
$$

However, according to the Biot-Savart law $[31,37]$ the magnetic field induced by each coil at the center $\mathrm{O}$ can be expressed in (6)-(8):

$$
\begin{aligned}
& \overrightarrow{B_{1}}=\frac{\mu_{0}^{2}}{4 R^{2}} \overrightarrow{i_{1}(t)} \\
& \overrightarrow{B_{2}}=\frac{\mu_{0}^{2}}{4 R^{2}} \overrightarrow{i_{2}(\mathrm{t})} \\
& \overrightarrow{B_{3}}=\frac{\mu_{0}^{2}}{4 R^{2}} \overrightarrow{i_{3}(\mathrm{t})}
\end{aligned}
$$

where $\mu_{0}$ is the vacuum permeability, $\mathrm{R}$ is the radius of the coil, and $i_{1}(t), i_{2}(t), i_{3}(t)$ are the current excitation in the first transmitter (Tx1), the second (Tx2), and the third (Tx3), respectively. Since the 
total magnetic field is the vector sum of the magnetic fields induced by all the coils, the total magnetic field induced at the center of the three orthogonal coils can be expressed as [31]:

$$
\vec{B}_{t}=\vec{B}_{1}+\vec{B}_{2}+\vec{B}_{3}
$$

\subsection{Design for Broadband Communication}

The power line communication is revived by establishing itself as one of the possible ways to ensure data communications in power grids. The latest development of the HomePlug Green PHY expresses the future trend, by reaching a communication speed of hundreds of Mbits per seconds [23,40]. Moreover, broadband over power line (BPL) physical layer uses multicarrier orthogonal frequency division multiplexing modulation carriers spaced at $24.414 \mathrm{kHz}$, with carriers from $2 \mathrm{MHz}$ to $30 \mathrm{MHz}$ to enable high throughput in a noisy environment. However, the IEEE 1901 and HomePlug AV2 standards extend this range to $50 \mathrm{MHz}$ and $86 \mathrm{MHz}$, respectively, achieving hundreds of Mbps [40-42]. Moreover, BPL offers a set of advantages that makes it an attractive complement to other RF wireless technologies, because it does not necessitate any new wiring installations, which considerably reduces the deployment costs and enables a new generation of high-speed indoor communications. Another advantage of BPL is that it can allow hard-to-reach nodes communication, where the RF signal suffers from high weakening, as in underground buildings, and low EMI infrastructure (hospitals).

The existing works performed in one-dimensional data transfer between the Tx and the Rx coils causes reduction in the data transfer during the misalignment between the coils. As detailed before, the omnidirectional power transfer can minimize the misalignment effects while improving the uniform power flow to the Rx. However, the full integration between three orthogonal coils for WPT and PLC could be a solution permitting the use of WPT without designing a new specification for data transmission on the same link providing a better solution for reliable, safe, and flexible power and data communication. However, the most important characteristic to be determined when evaluating the possibility of using a channel for data communication is the theoretical channel capacity calculated as a function of the coupling coefficient of the WPT system, in the presence of additive white Gaussian noise. The performance of the 3-D coil WPT channel in handling a high date rate communication speed is evaluated by estimating the channel capacity $(C)$, based on the Shannon-Harltey approach. In fact, the modulated data signal which is transmitted and/or the receiver is filtered by the bandpass channel transfer's function, $H(f)$, is corrupted by the noise and affected by the harmonic distortions generated by the nonlinear electrical circuit used at the WPT transmitter (e.g., RF power amplifier) and receivers (e.g., rectifier) $[23,24,26]$ given by:

$$
C=\int_{0}^{B W} \log _{2}\left(1+\left(\frac{S(f)}{N(f)}\right)\right) d f
$$

where $C$ is the channel capacity in bits per second (bps), $B W$ is the bandwidth of the channel, $S(f)$ and $(f)$ are the signal and the noise power spectrum, respectively. It is worth noting that some approximations are considered during the determination of this figure of merit. In fact, the injected power and noise signals spectrum, are frequency independent (i.e., $(f)=S I$ and $(f)=N$ ) over the working frequency band. Moreover, the noise is considered as an additive white Gaussian noise at the receiver. Therefore, the signal to noise ratio (SNR) is then defined as $S N R=S I / N$, with a frequency band [0-100] MHz, and the received power spectrum can be expressed as a function of the injected power spectrum and the transfer function $[23,26]$ given by:

$$
S(f)=|H(f)|^{2} S_{I}
$$

The channel capacity linearly depends on the WRIC bandwidth, $B W$, and nonlinearly depends on the attenuation of the power and/or data channel transfer function $H|(f)|$. Moreover, $H|(f)|$ and BW both 
depend on the misalignment angle $\theta$ and coupling coefficients, $k\left[k_{1 R}, k_{2 R}, k_{3 R}\right]$. Accordingly, (11) can be rewritten as:

$$
C=\int_{0}^{B W} \log _{2}\left(1+|H(f, k, \theta)|^{2} * S N R\right) d f
$$

The complex-valued transmitting coefficient, $S_{21}(f)$, of two-port inductive channel can be written [24,27]:

$$
S_{21}(f)=\frac{2 \cdot V_{L}(f)}{V_{s}(f)} \cdot \sqrt{\frac{R s}{R_{L}}}=2 \cdot H(f) \cdot \sqrt{\frac{R s}{R_{L}}}
$$

\section{Current Omnidirectional WPT Prototypes}

In the domain of consumer electronics, the wireless charger is becoming prevalent, and several WPT standards have been set up to boost the interoperability of different chargers for high power applications such as electrical vehicles [7,11,17], chargers, and grid interfaces. The WPT technique improves the devices' safety and reduces the complexity of connectivity. WPT was used to rapidly recharge electric vehicle batteries to meet the extended working range. Battery charging can be done during a temporary stop in stations based on receiving and transmitting coils, through the transmitting coil embedded in the roadway and receiving coil equipped on the bus or rail transit. Some of these applications have been launched into the market, while others are not ready yet for the commercial market. For instance, automotive charging is an innovation, but it is still unavailable in the market because of the need to standardize its charging infrastructure. The latest iPhone 8 , iPhone $X$, and Samsung have installed wireless charging chips, which enhance their ability to hold a charge. Moreover, among the existing omnidirectional WPT system we can mention the wireless power bowl demonstrated by Intel [31] and shown in Figure 2.

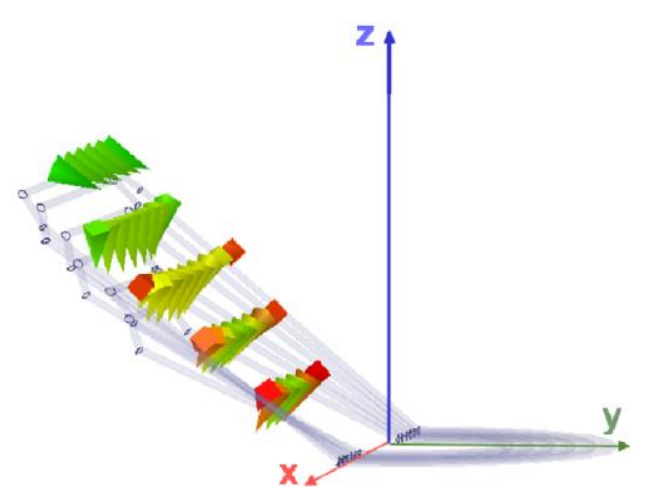

(a)

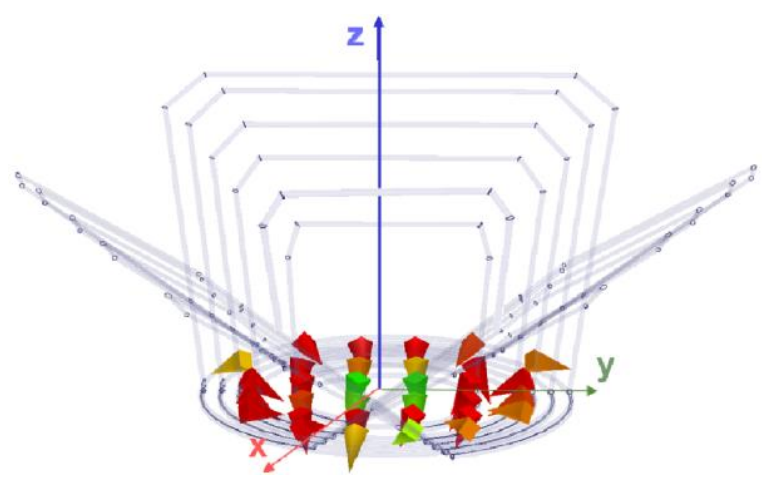

(b)

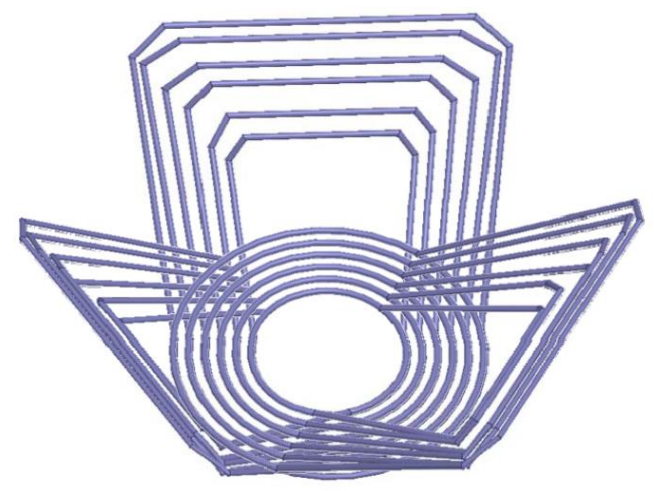

(c)

Figure 2. Magnetic field distribution in Intel's charging prototype [31]: (a) the side face; (b) the bottom; (c) transmitter coils arrangement. 
In fact, this charging bowl has more than one direction of magnetic field distribution: vertical field in the bottom center and perpendicular field in the side face as detailed in Figure 2. The planar device can be charged efficiently when it lies on the side face. However, in the bottom of the bowl, there is no omnidirectional field. Therefore, there is no omnidirectional wireless power transfer capability with Intel's charging bowl prototype. Moreover, the use of identical current excitation (i.e., identical current control) does not generate a magnetic field vector that points in all directions in a 3-D manner, which is an indispensable feature for a real omnidirectional wireless power transfer. For genuine omnidirectional wireless power transfer, omnidirectional WPT platform with three orthogonal symmetric coils was developed by R.R.Y.HUI's research group in the university of Hong Kong [34,35,37] as shown in Figure 3.
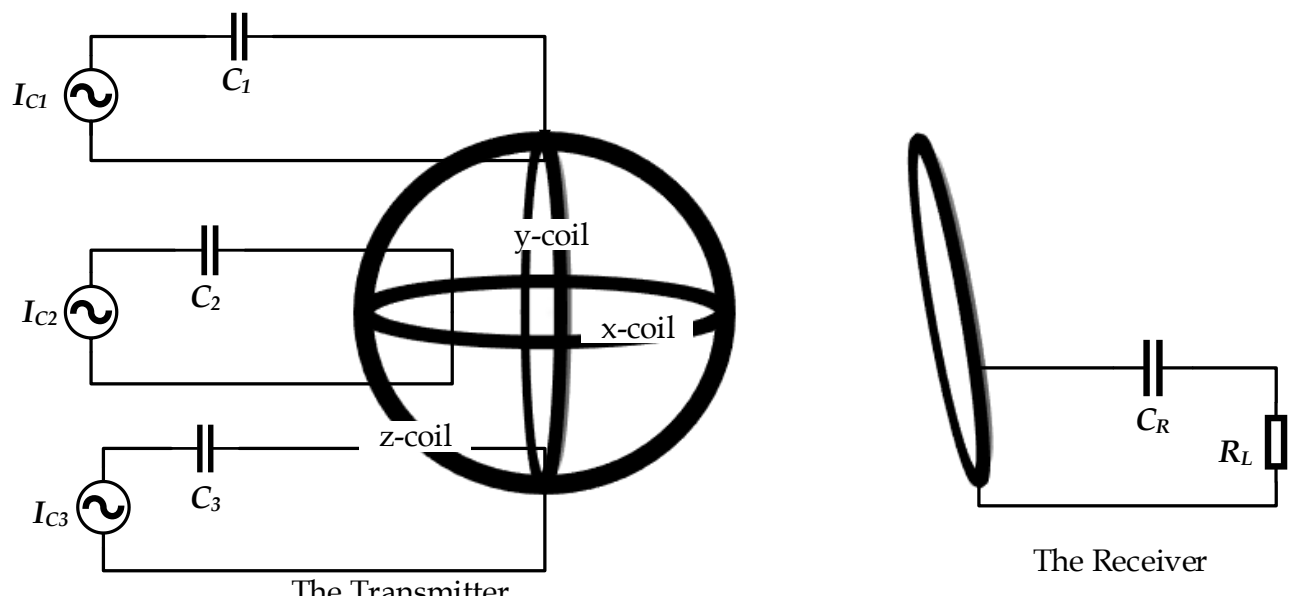

The Receiver

Figure 3. Three-orthogonal-coil structure for omnidirectional WPT [37].

The proposed omnidirectional WPT system consists of three orthogonal transmitter coils and a planar receiver coil. However, since the total magnetic field is the vector sum of the magnetic fields induced by all the coils, it is impossible to achieve an omnidirectional WPT by fixing the total magnetic field at a particular direction. However, it is required for the orthogonal coils current to be non-identical.

Deferent control techniques are used in 3-D transmitters to steer the direction of the synthetic magnetic field depending on the position of the Rx [43], and achieving rotating magnetic field vectors such as the current amplitude modulation and phase angle control or frequency modulation as detailed in [43].

\section{Characterization of Omnidirectional WPT Transceivers via Resonant Inductive Coupling}

\subsection{Magnetic Fields Distribution Finite Element Simulation}

As detailed in $[39,44]$ the square coil has magnetic flux field, which is larger than the circular coil when the same diameter length is occupied, engendering a stronger magnetic field strength, which has a positive result on the transfer power and transfer efficiency, and enhances the space utilization rate. Since the square spiral has right angles, current changes abruptly at the right angle of the coil, and the resistance is large. However, in [44] the authors combined the benefits of circular and square coils by replacing the right angle of the spiral square coils with rounded corners, thus rounded square coil is selected as the coils' shape.

The finite element modeling and simulation of 3-D WPT coil system are evaluated through the Multiphysics software. The AC-DC physics model with magnetic field module is selected for analysis in the frequency domain study at different coupling points. To interface the resonant circuit and for the coil excitation, an electric circuit is required with the AC-DC module in further simulation. In these analyses, only the coils constituting the device under test are considered without any additional 
equipment. The relative placement of transmitting and receiving coils which are used in the simulation, is shown in Figure 4.

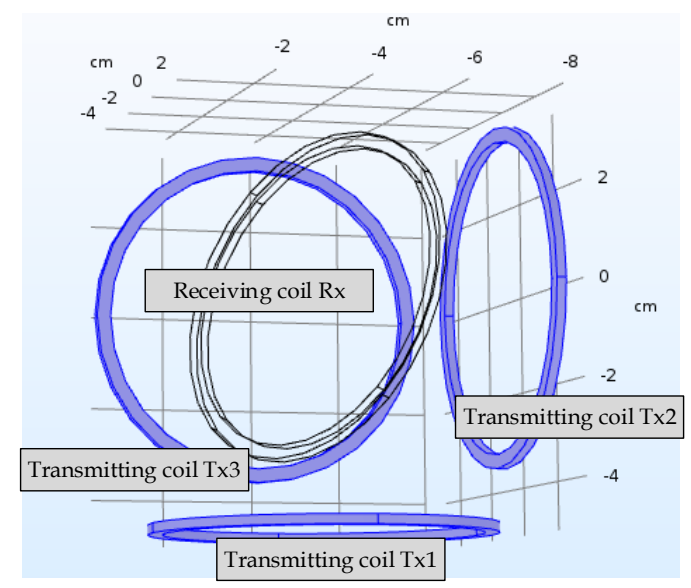

Figure 4. Simulation geometry of three orthogonal coils for WPT.

The transmitting and receiving coils' model parameters are consistent. The coils are composed of copper wire. The radius of each coil is $r_{1}=r_{2}=3.6 \mathrm{~cm}$. The number of turns $N_{1}=N_{2}=1$. The transmitting coils are located in the $X$ plane, $Y$ plane, and $Z$ plane. The excitation current of the input port is $1 \mathrm{~A}$ and $\theta$ is the deviation angle between the transmitting and the receiving coils.

The magnetic field distribution and magnetic flux density are attained at different coupling points. The results shown in Figure 5 illustrate the obtained magnetic flux density and magnetic field distribution are almost equal under the three different alignment conditions.

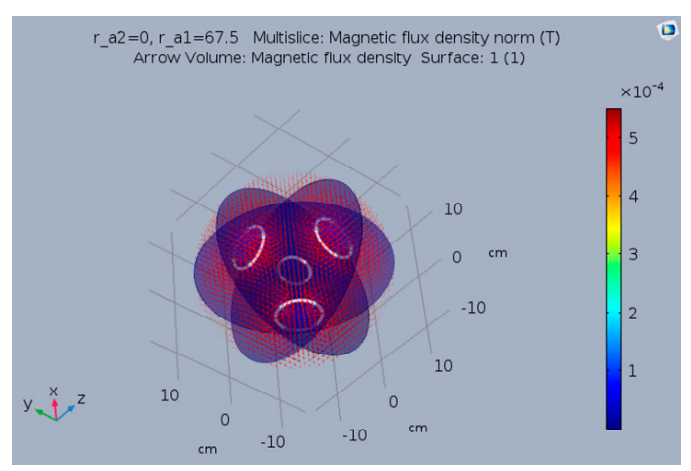

(a)

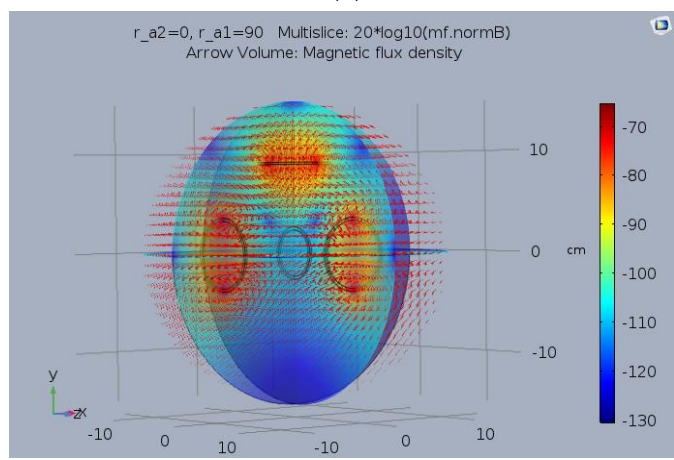

(c)

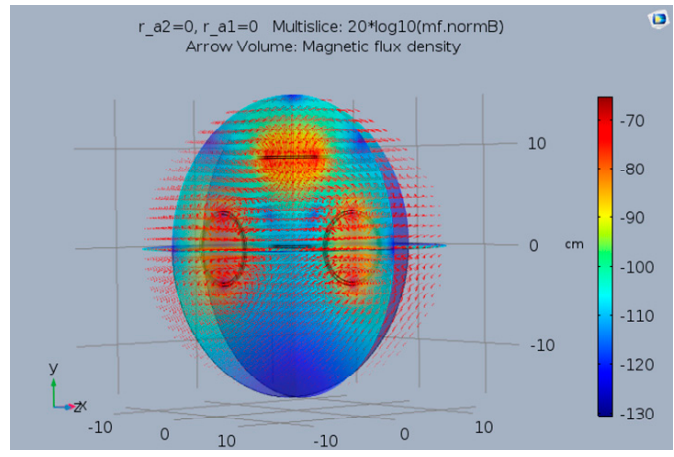

(b)

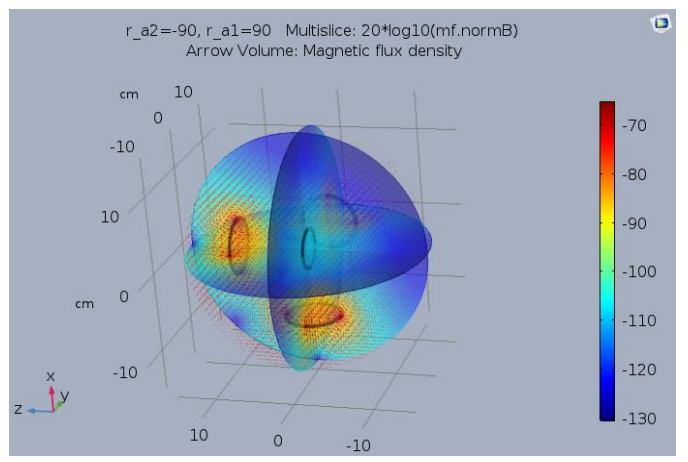

(d)

Figure 5. Plot of magnetic flux density while the receiving antenna is rotating from 0 to 90 and 0 to -90 degrees with a step of 22.5 degrees. (a) The magnetic field distribution; (b) Rx parallel to x-coil. (c) Rx parallel to y-coil. (d) Rx parallel to z-coil. 
As the Tx and Rx coils are in the same plane, the power transfer to the $\mathrm{Rx}$ is maximized. The orientation in the Rx coil is analyzed by changing the rotation angle of the Rx-coil, as presented in Figure 5 .

For all possible angle numbers, the Rx receives more magnetic field intensity with respect to the TX coils. Hence, the misalignment effect can be minimized by designing three-dimensional TX coil structure, as the Tx and Rx coils are in the same plane the power transfer to the Rx is maximized. The orientation in the Rx coil is analyzed by changing the rotation angle of the Rx-coil, as presented in Figure 5.

\subsection{Dependency on the Coupling Coefficients $k_{1 r}, k_{2 r}, k_{3 r}$ and the Misalignment Angle}

The multiport $S$-parameters analysis is considered to evaluate the power links as shown in Figure 6.

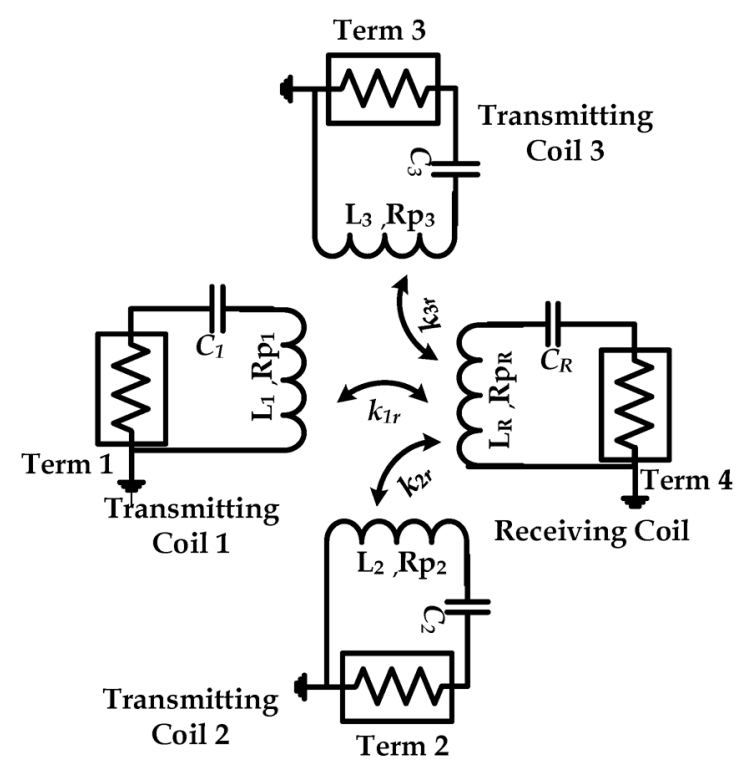

Figure 6. Multiport S-parameters simulation setup used to characterize the combined WPT and data communication.

For the proposed three orthogonal TX, the Rx coil equivalent circuit is designed using Advanced System Design (ADS) software for the S-parameter analysis. The mutual coupling between the Tx and $\mathrm{Rx}$ coil changes for the different orientations of the $\mathrm{Rx}$ coil $M_{i j}=\mathrm{k}_{\mathrm{ij}} \sqrt{L_{i} L_{j}}$. In Figure 6 the power link is represented by the port terminals 1 and 4 for the first transmitter. The port terminals 2 and 4 for the second transmitter and port terminals 3 and 4 for the third transmitter. The values of the Tx and Rx electronic components (inductance, capacitance, and resistance) are displayed in Table 1.

Table 1. Circuit component and parameter values of the simulation setup.

\begin{tabular}{cc}
\hline Parameters & Values \\
\hline$R_{s}=R_{L}$ & $50.0 \Omega$ \\
\hline$L_{1}=L_{2}=L_{3}=L_{\mathrm{R}}$ & $1.0 \mu \mathrm{H}$ \\
\hline$C_{1}=C_{2}=C_{3}=C_{\mathrm{R}}$ & $235 \mathrm{pF}$ \\
\hline$R_{p 1}=R_{p 2}=R_{p 2}=R_{p \mathrm{R}}$ & $0.15 \Omega$ \\
\hline$k_{1 \mathrm{R}}, k_{2 \mathrm{R}}, k_{3 \mathrm{R}}$ & $10^{-3}$ to 1 \\
\hline
\end{tabular}

Since, the coupling coefficient depends on the distance and the misalignment angle $\theta$ between the transceivers, it is instructive to analyze the efficiency of the proposed system as a function of these 
variables considering the planar structures with single transmitter coil $\mathrm{Tx}$ and single receiver coil $\mathrm{Rx}$ as shown in Figure 7.

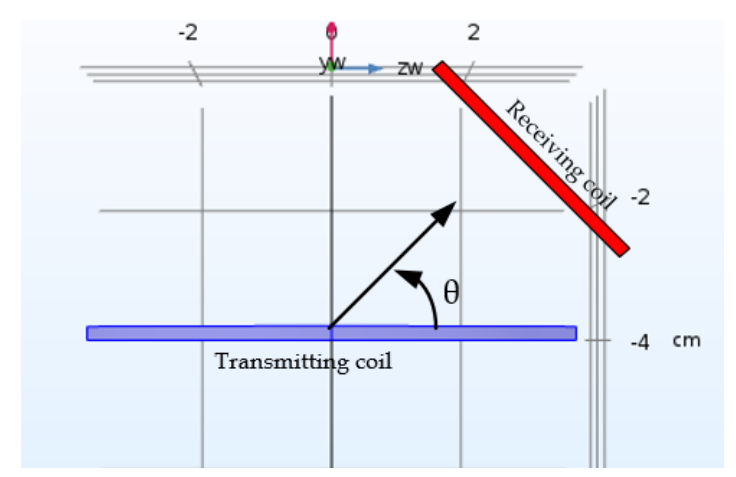

Figure 7. Geometry model of the single-receiver system.

The power efficiency can be expressed [33]:

$$
\eta=\frac{P_{\text {out }}}{P_{\text {in }}}=\frac{R_{L} w^{2} M_{\text {max }}^{2} \sin (\theta)}{\left(R_{L}+R_{p 2}\right)^{2}\left(R_{S}+R_{p 1}\right)+\left(R_{L}+R_{p 2}\right) w^{2} M_{m a x}^{2} \sin (\theta)}
$$

where $\theta=[0, \pi], M_{\max }$ is maximal mutual inductance between the two coils and can be computed by (15) in which $\mu$ is air permeability, $N_{1}$ and $R_{1}$ are the number of turns and radius of the transmitting coil, $N_{2}$ and $R_{2}$ are the number of turns and radius of the receiving coil, respectively, and $d_{s 1}$ is the distance between the centers of the two coils given by [33]:

$$
M_{\max }=\frac{\pi \mu N_{1} N_{2} R_{1}^{2} R_{2}^{2}}{2\left(r_{1}^{2}+d_{s 1}^{2}\right)^{3 / 2}}
$$

The transfer efficiency (14) as a function of the deviation angle $\theta$ values for single-receiver WPT system is reported in Figure 8.

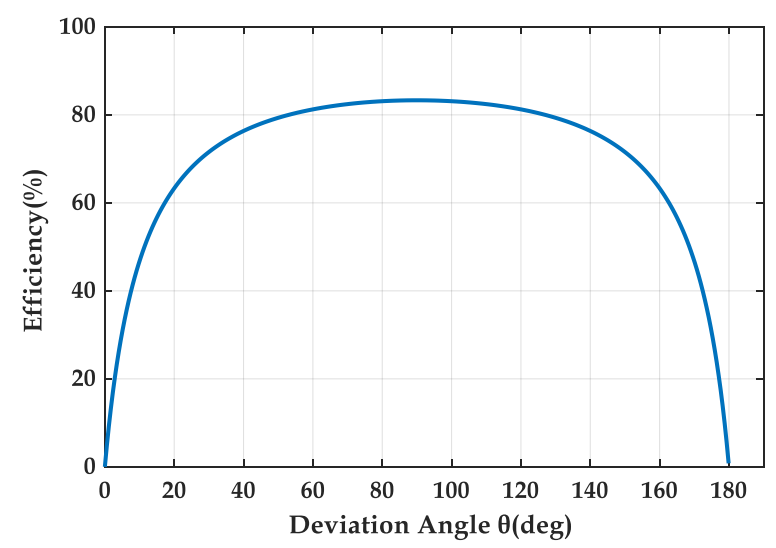

Figure 8. Transfer efficiency as a function of the deviation angle $\theta=[0, \pi]$.

The power transfer efficiency is affected by the deviation angle. However, a concomitant decline in the transfer efficiency can be observed with the value of $\theta$ and the maximum transfer efficiency value is reached for $\theta=\pi / 2$ which means that the two coils are perfectly aligned. In fact, the analysis results indicate that the misalignment angle significantly impacts the power transfer efficiency, since, 
the system performances depend on the receiver's positions, which reduce the receiver working flexibility when being charged.

\subsection{Dependency on the Coupling Coefficients $k_{1 R}, k_{2 R}, k_{3 R}$ and the Misalignment Angle}

This subsection demonstrates the dependency of the proposed system performance on the coupling coefficients $k_{1 R}, k_{2 R}$, and $k_{3 R}$ respectively. Considering:

- $\quad S_{1,4}(f), S_{(T x 1, R x)}$ represent the transmission gain between the first transmitter (Tx1) and the receiver (Rx) with the coupling coefficient $k_{1 R}$.

- $\quad S_{2,4}(f), S_{(T x 2, R x)}$ represent the transmission gain between the first transmitter (Tx1) and the receiver ( $\mathrm{Rx})$ with the coupling coefficient $k_{2 R}$.

- $\quad S_{3,4}(f), S_{(T x 3, R x)}$ represent the transmission gain between the first transmitter (Tx1) and the receiver $(\mathrm{Rx})$ with the coupling coefficient $k_{3 R}$.

First, the coupling between the second and third transmitting coils with Rx-coil are fixed at 0.09 $\left(k_{2 R}=k_{3 R}=0.09\right)$ while sweeping the coupling between the Tx1 and Rx coils $k_{1 R}=[0.001,0.3,0.6,0.9]$. The $S$-parameter simulation results are shown in Figure 9.

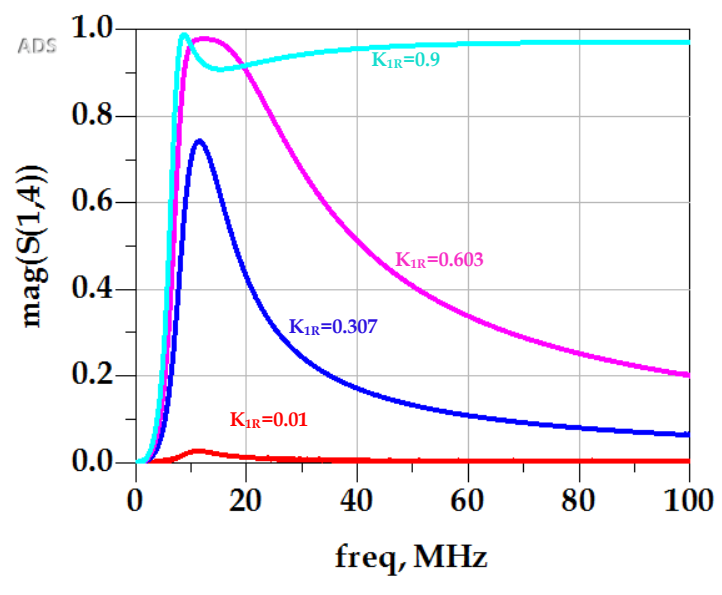

(a)

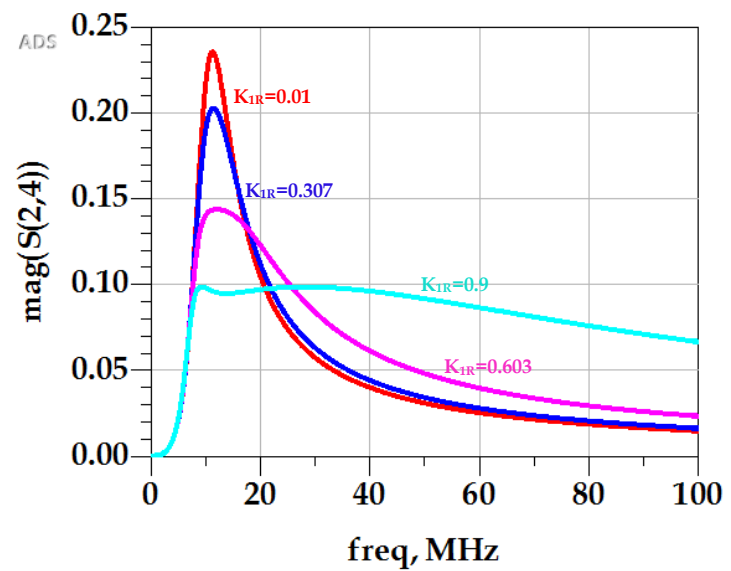

(b)

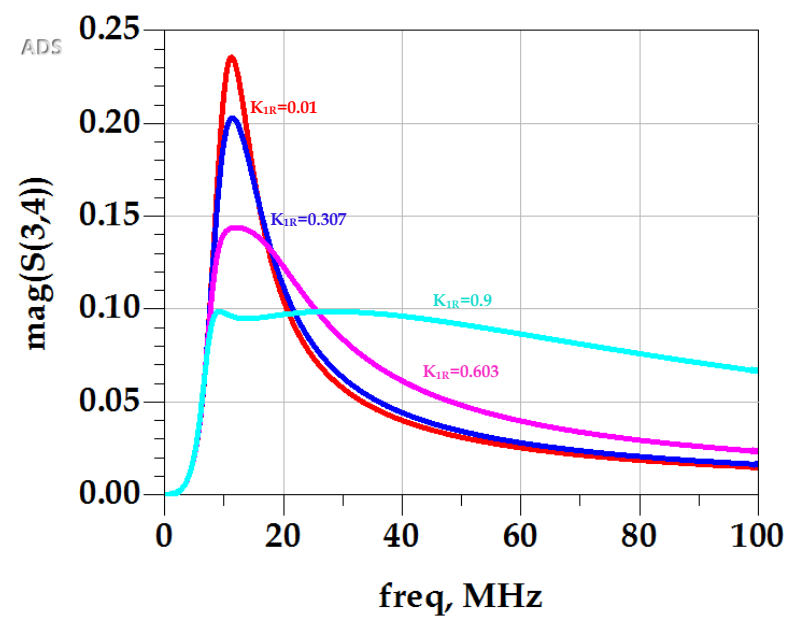

(c)

Figure 9. S parameter simulation results: (a) $\mathrm{S}(\mathrm{Tx} 1, \mathrm{Rx})$; (b) $\mathrm{S}(\mathrm{T} x 3, \mathrm{Rx})$; (c) $\mathrm{S}$ (Tx2, $\mathrm{Rx}$ ), for different $\mathrm{k}_{1 \mathrm{R}}$ at $\mathrm{k}_{2 \mathrm{R}}=\mathrm{k}_{3 \mathrm{R}}=0.09$ in the frequency band freq $[0,100] \mathrm{MHz}$.

Figure $9 b, c$ shows that maximum transmission gain is reached even for lower coupling coefficients values (e.g., $\left.k_{1 R}=0.01\right)$ from $\mathrm{Tx} 2$ and $\mathrm{Tx} 3$. $\left(S_{2,4}(f)=S_{3,4}(f)=0.235\right)$. However, as $k_{1 R}$ increases 
as the maximum transferred power is reached from Tx1 as reported in Figure 9a. Consequently, as $k_{1 R}$ increases as the Rx coil is perfectly aligned with Tx1 and maximum power transfer is obtained $S_{1,4}(f)=0.97$ for $k_{1 R}=0.9$. Next, the coupling between the Tx1-Rx and Tx3-Rx are increased to be 0.5 $\left(k_{1 R}=k_{3 R}=0.5\right)$ while varying $k_{2 R}=[0.01,0.3,0.6,0.9]$ as shown in Figure 10.

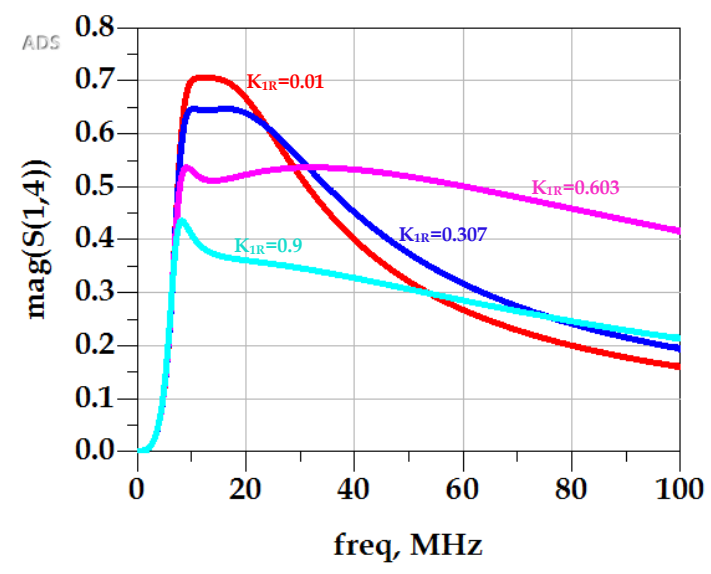

(a)

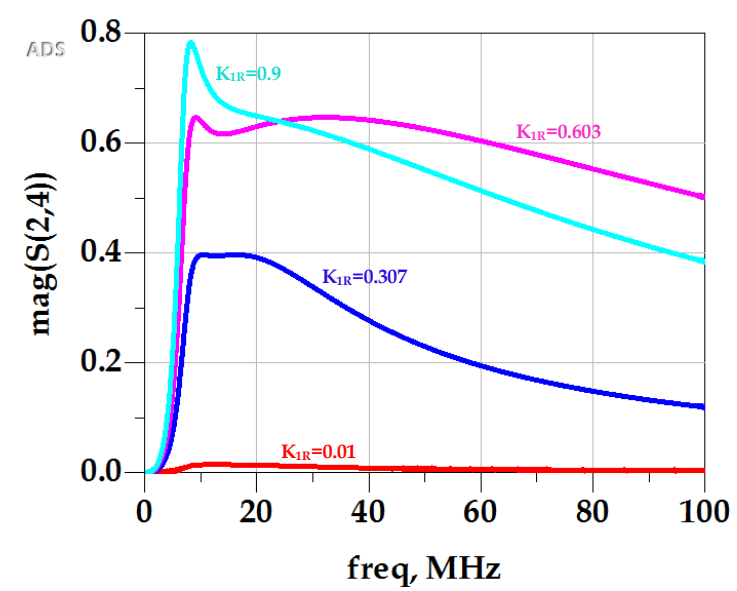

(b)

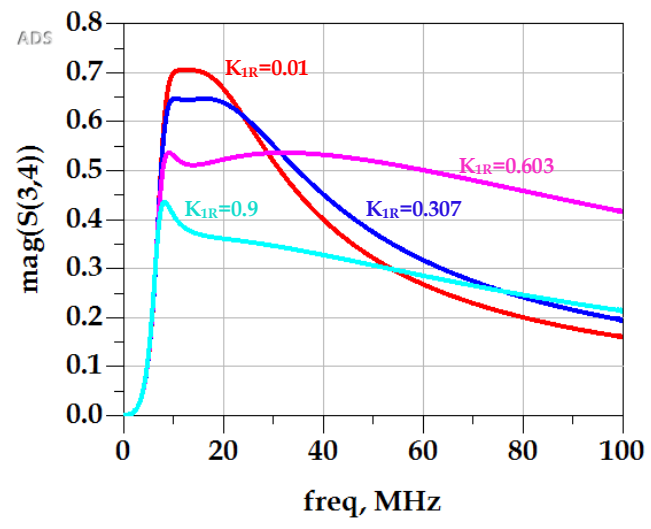

(c)

Figure 10. $S$ parameter simulation results: (a) $S(T x 1, R x)$; (b) $S(T x 3, R x)$; (c) $S(T x 2, R x)$ for different $k_{2 R}$ at $k_{1 R}=k_{3 R}=0.5$ in the frequency band freq $[0,100] \mathrm{MHz}$.

Similarly, for the Tx2-Rx as the coupling coefficient $k_{2 R}$ increases, the maximum transferred power is obtained from $\mathrm{T} \times 2$ as reported in Figure $10 \mathrm{~b} S_{2,4}(f)=0.97$ for $k_{2 R}=0.9$. In addition to that, better value for the transmission gain is obtained when $k_{1 R}=k_{2 R}=0.5$, from Tx3, respectively, as shown in Figure 10a,b $S_{1,4}(f)=S_{3,4}(f)=0.7$ for $k_{2 R}=0.01$. Finally, the coupling coefficients between Tx1-Rx and Tx2-Rx are constant and equal to $0.2\left(k_{1 R}=k_{2 R}=0.2\right)$ while changing $k_{3 R}=0.01,0.3,0.6,0.9$ as shown in Figure 11.

The reported results in Figure 11 show that a maximum transmission gain is reached even for lower coupling coefficient $k_{3 R}$ values from Tx1, Tx2, respectively, $S_{1,4}(f)=S_{2,4}(f)=0.39$ for $k_{3 R}=0.001$ at the designed resonant frequency as illustrated in Figure 11a,b, in fact, as $k_{3 R}$ increases as the value of $S_{1,4}(f)$ and $S_{2,4}(f)$ decreases.

Therefore, the transceivers enjoy a wide bandwidth channel with small attenuation while taking full advantage of the power efficiency of the WPT channel. The power channel is not very selective for higher values of $k_{1 R}, k_{2 R}$ and $k_{3 R}$, this enables the simultaneous WPT and data communication between WPT terminals. Besides, as the coupling coefficients increase, which can be practically done by diminishing the distance and the misalignment angle between the three transmitting and receiving coils, a maximum transferred power and a wide bandwidth for data communication, which guarantee 
maximum channel capacity through the resonant inductive channel, are achieved. It is also noticeable that the channel bandwidth BW can be used to simultaneously transfer power and communicate data between the power sources and receivers given the possibility to modulate the power signal with the baseband data signal. Hence, for the three-dimensional orthogonal structure the Rx coil will have better power transfer from any of the Tx coils regardless of the orientation.

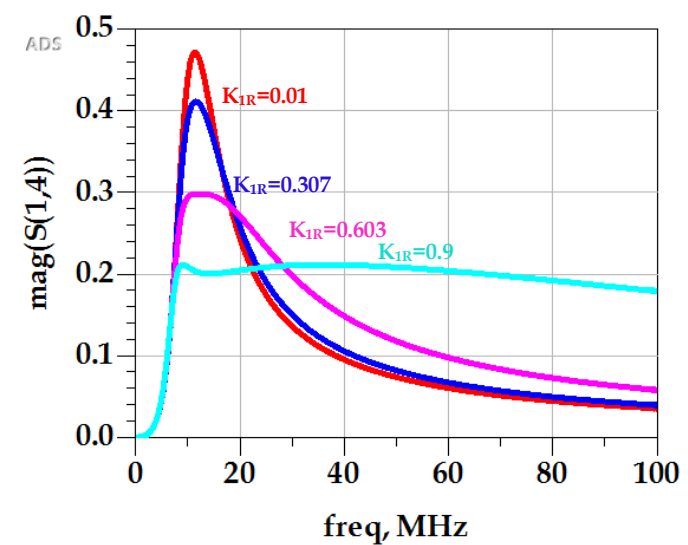

(a)

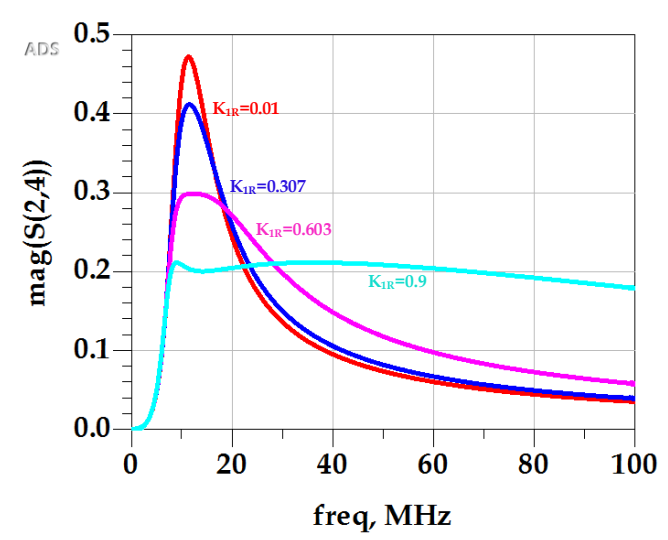

(b)

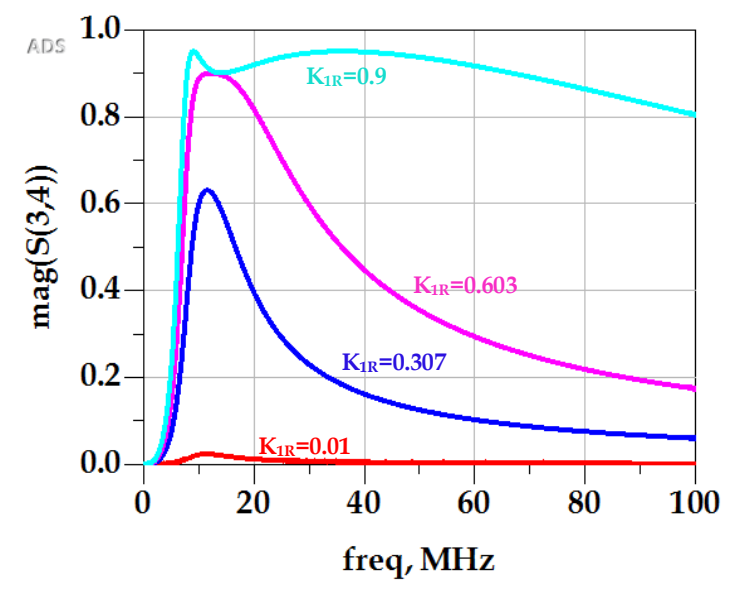

(c)

Figure 11. $\mathrm{S}$ parameter simulation results (a) $\mathrm{S}(\mathrm{T} x 3, \mathrm{Rx})$; (b) $\mathrm{S}(\mathrm{T} x 1, \mathrm{Rx})$; (c) $\mathrm{S}(\mathrm{T} x 2, \mathrm{Rx})$ for different $k_{3 R}$ at $k_{1 R}=k_{2 R}=0.2$ in the frequency band freq $[0,100] \mathrm{MHz}$.

Moreover, the variation of the maximum value of $\left|S_{1,4}(f)\right|,\left|S_{2,4}(f)\right|$ and $\left|S_{3,4}(f)\right|$ as a function of $k_{1 R}, k_{2 R}$, and $k_{3 R}$, respectively, are shown in Figure 12.

Firstly, as the coupling between the Tx1-Rx increases, the Rx coil is perfectly aligned and the distance is reduced with the first transmitter. However, maximum transferred power is obtained, as shown in Figure 12a. Similarly, the power links of both Tx2-Rx and Tx3-Rx saturate for higher values of the coupling factors $k_{2 r}, k_{3 r}$ (i.e., $k_{2 R}=k_{3 R} \geq 0.6$ ) as illustrated in Figure 12b,c. In fact, $\left|S_{2,4}(f)\right|$, $\left|S_{3,4}(f)\right|$ decrease as a function of $k_{1 R}$ reaching its maximum for lower $k_{1 R}$ values. Likewise, Figure $12 \mathrm{~b}$ shows that as the coupling coefficient between Tx2 and Rx increases, a maximum transferred power is reached from Tx2 to $\mathrm{Rx}$, in this case $\left|S_{1,4}(f)\right|,\left|S_{3,4}(f)\right|$ decrease as a function of $k_{2 R}$. Similarly, for Figure 12c the maximum received gain is reached from Tx3 while increasing $k_{3 R}$.

In all the cases, the obtained results show that the power transfer efficiency is maximum when the Rx coil is impeccably aligned with one of the three transmitters. 


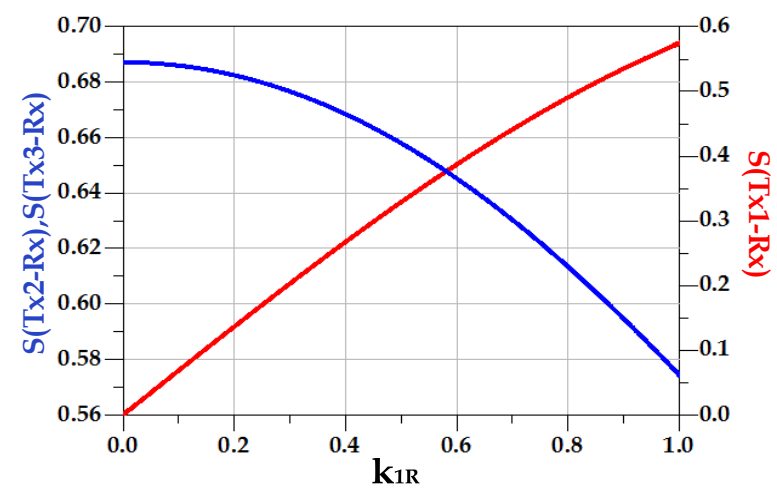

(a)

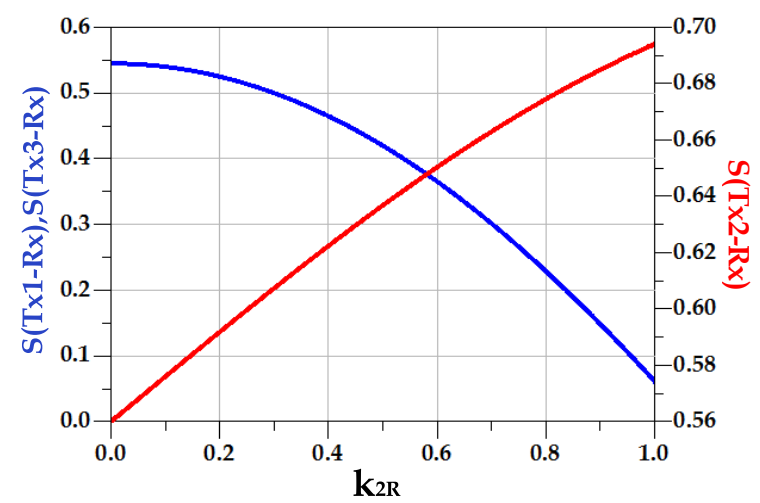

(b)

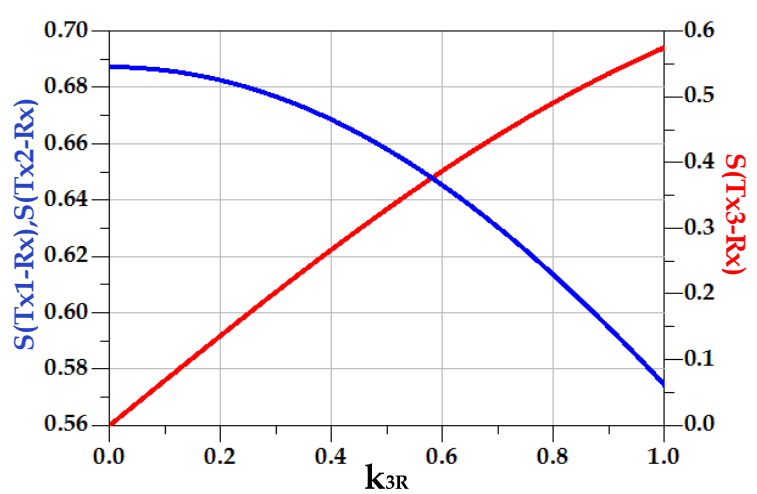

(c)

Figure 12. Maximum of $\mathrm{S}(\mathrm{Tx} 1, \mathrm{Rx}), \mathrm{S}(\mathrm{Tx} 2, \mathrm{Rx})$, and $\mathrm{S}(\mathrm{Tx} 3, \mathrm{Rx}) .(\mathbf{a}) k_{1 R} ;(\mathbf{b}) k_{2 R} ;$ (c) $k_{3 R}$.

\subsection{Channel Capacity Evaluation}

The theoretical channel capacity in the frequency band [1-100] $\mathrm{MHz}$ is assessed by using the Shannon-Hartley (12). The dependency of the channel capacity on the coupling coefficients $k_{1 R}, k_{2 R}$, and $k_{3 R}$ is evaluated and the simulation results are illustrated in Figures 13-15.

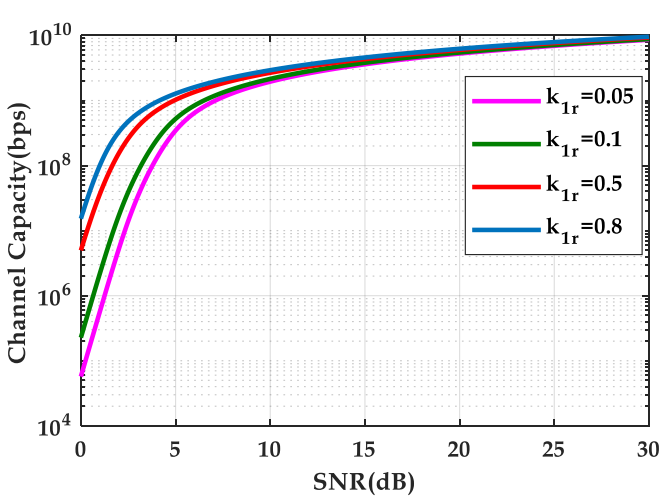

(a)

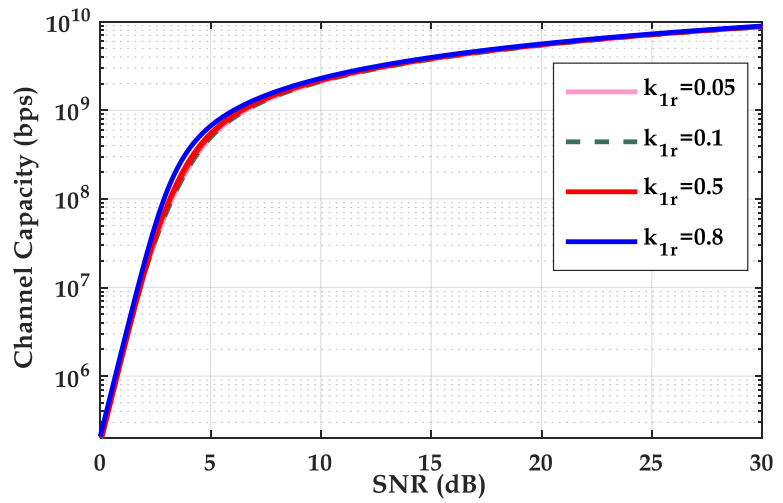

(b)

Figure 13. Channel capacity of (a) Tx1-Rx; (b) Tx2-Rx, Tx3-Rx as a function of the SNR (dB), for different $k_{1 R}$ values, at $k_{2 R}=k_{3 R}=0.09$. 


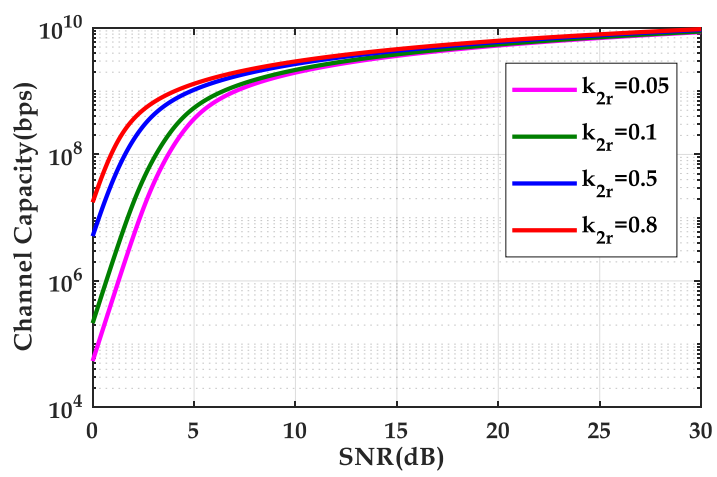

(a)

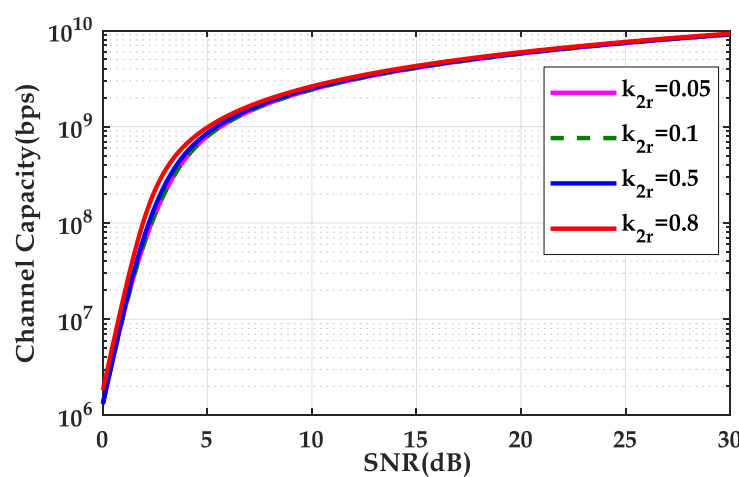

(b)

Figure 14. Channel capacity of, (a) Tx2-Rx; (b) Tx1-Rx, Tx3-Rx as a function of the SNR (dB) for different $k_{2 R}$ values at $k_{1 R}=k_{3 R}=0.25$.

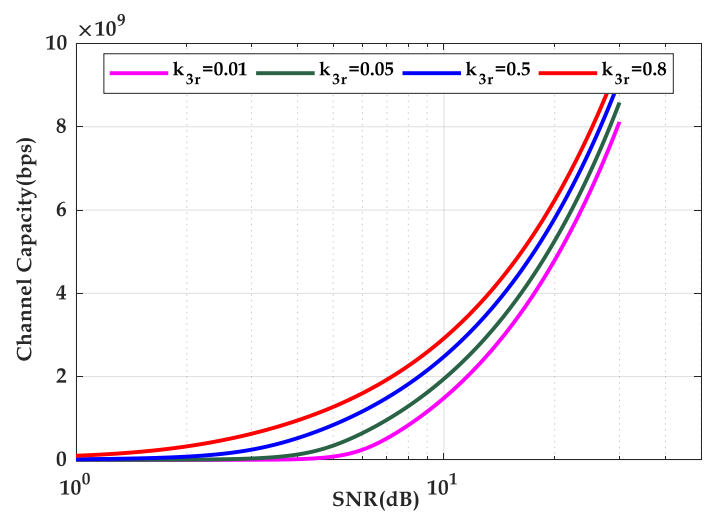

(a)

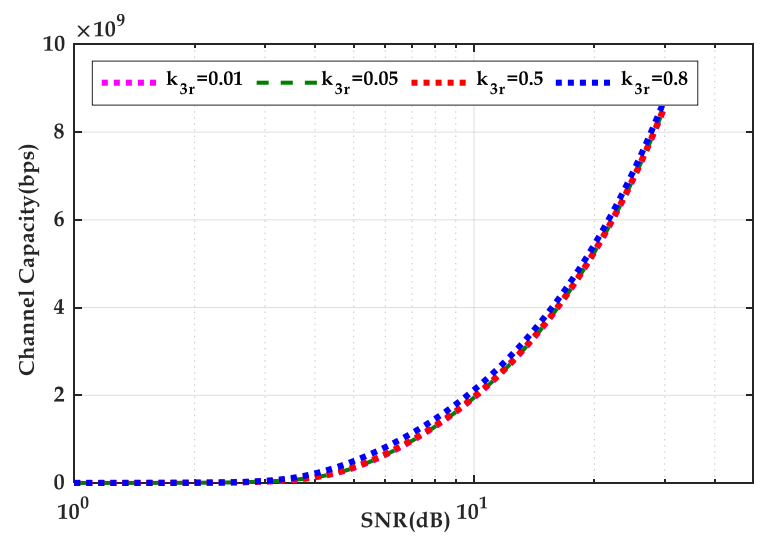

(b)

Figure 15. Channel capacity of (a) Tx3-Rx; (b) Tx1-Rx, Tx2-Rx as a function of the SNR (dB) for different $k_{3 R}$ values at $k_{1 R}=k_{2 R}=0.05$.

As confirmed, the coupling coefficients $\left(k_{1 R}, k_{2 R}, k_{3 R}\right)$ increase, and the maximum data rate is attained in all scenarios. Likewise, the channel capacity of data transfer can be maximized with high SNR value, in fact, as the $S N R$ is lower than $5 \mathrm{~dB}$, the channel capacity is highly reliant on the coupling coefficients between the transmitting and receiving coils, and linearly increases as a function of the SNR. It is noteworthy that in spite of the relatively low bandwidth of the channel, a high channel capacity can be achieved, under a reasonable noise scenario. In addition, the theoretical channel capacity does not increase significantly with the variation of the coupling coefficients, permitting an efficient data transfer between the power grid and the vehicle when it is charged by a WPT system. However, the behavior of the PLC system is estimated to be stable.

The proposed 3-D structure offers better power and data transfer efficiency regardless of the Rx coil orientation achieving maximum data rate. For instance, a detailed comparison of the Cmin and Cmax, which are the minimum and the maximum values of the channel capacity for both Tx1-Rx and Tx2/Tx3-Rx, respectively, is shown in Figure 13 and reported in Table 2. 
Table 2. Comparison between the minimum and the maximum values of the data rate, for different $k_{1 R}$ as $\mathrm{SNR} \in[1,30] \mathrm{dB}$.

\begin{tabular}{ccccc}
\hline & \multicolumn{2}{c}{ Tx1-Rx } & \multicolumn{2}{c}{ Tx2-Rx/Tx3-Rx } \\
\hline & $\mathrm{SNR}=0 \mathrm{~dB}$ & $\mathrm{SNR}=30 \mathrm{~dB}$ & $\mathrm{SNR}=0 \mathrm{~dB}$ & $\mathrm{SNR}=30 \mathrm{~dB}$ \\
\hline & $C_{\min }(\mathrm{bps})$ & $C_{\max }(\mathrm{bps})$ & $C_{\min }(\mathrm{bps})$ & $C_{\max }(\mathrm{bps})$ \\
\hline$k_{1 R}=0.05$ & $5.717110^{4}$ & $8.585210^{9}$ & $1.847610^{5}$ & $8.754710^{9}$ \\
\hline$k_{1 R}=0.5$ & $4.964310^{6}$ & $9.308510^{9}$ & $1.696810^{5}$ & $8.813910^{9}$ \\
\hline
\end{tabular}

\section{Conclusions}

Designing an integrated WPT-PLC for UAVs reduces the devices' complexity and manufacturing costs. However, the design of a maximum power transfer and high data rate communication between the power and data transmitters and receivers through the wireless inductive channel can face a challenging bandwidth and misalignment. This contribution has presented the analysis of a 3-D omnidirectional wireless-coil inductive channel for both WPT and data communication. The design challenges were investigated and the simulation results of multiport $S$-parameters setup were discussed while considering the maximum power transfer and channel capacity performances. Moreover, the analysis carried out in this work demonstrates the feasibility of the use of 3-dimensional WPT systems for data communication, achieving theoretical capacities from tens of Kbit/s to few Gbit/s depending on the SNR value and the distances between the transceivers.

The coexistence of a narrowband channel for power transfer, and a broadband channel for data communication, using the same coils is feasible thanks to the 3-dimensional omnidirectional wireless inductive coupling, which allows two existing technologies, WPT and PLC, to share the same channel while achieving maximum transferred power and better data rate. However, compared with the directional WPT, which is limited by the deviation angle, the omnidirectional wireless power transfer (WPT) system offers great flexibility in free-positioning of the loads in a 3-dimensional manner.

As a future work, the system performance can be characterized in more detail with a study on the importance of the decoupling filters in order to enable separated data and power transfer and prevent the interference between the high power signal and low power data signal. Moreover, the experimental setup and results will be performed in further study, taking into consideration the requirements of such integrated system, in order to guarantee an efficient power transfer and a correct data transmission.

Author Contributions: S.Z., W.D., R.M., and H.B. conceived the formulation, performed the simulations, analyzed the simulation results, and wrote the paper. All authors have read and agreed to the published version of the manuscript.

Funding: This research received no external funding.

Acknowledgments: Ministry of Higher Education and Scientific Research, Republic of Tunisia; through Foundation for Science and Technology (FCT) under the ICT (Institute of Earth Sciences) project UIDB/04683/2020; Portuguese Funds through the Foundation for Science and Technology (FCT) under the LAETA project UIDB/50022/2020.

Conflicts of Interest: The authors declare no conflict of interest.

\section{List of Symbols}

Q Quality factor

$M \quad$ The mutual inductance

$k \quad$ The coupling coefficient

$\mu_{0} \quad$ Vacuum permeability

$r \quad$ Radius of the coil

$N \quad$ Number of turns of the coil

$B \quad$ Magnetic field

C Channel capacity

$R_{L} \quad$ Load resistance 


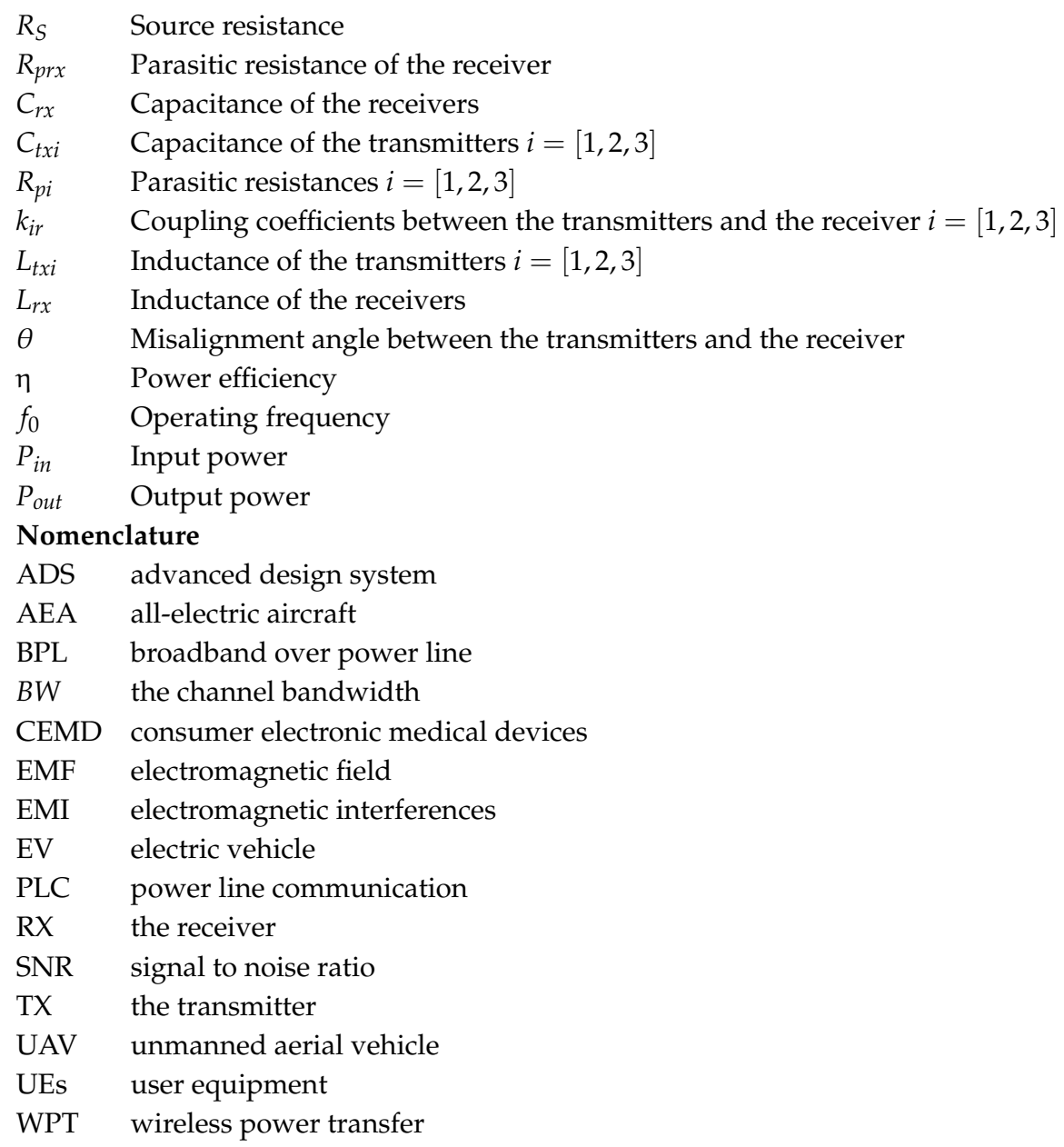

\section{References}

1. Melício, R.; Mendes, V.M.F.; Catalão, J.P. Modeling and Simulation of Wind Energy Systems with Matrix and Multilevel Power Converters. IEEE Lat. Am. Trans. 2009, 7, 78-84. [CrossRef]

2. Seixas, M.; Melicio, R.; Mendes, V.M.F. Fifth harmonic and sag impact with a balancing new strategy for capacitor voltages. Energy Convers. Manag. 2014, 79, 721-730. [CrossRef]

3. Batista, N.C.; Melicio, R.; Matias, J.C.O.; Catalão, J.P.S. ZigBee standard in the creation of wireless networks for advanced metering infrastructures. In Proceedings of the 16th IEEE Mediterranean Electrotechnical Conference (MELECON), Yasmine Hammamet, Tunisia, 25-28 March 2012; pp. 220-223.

4. Gomes, I.; Pousinho, H.; Melíco, R.; Mendes, V. Bidding and Optimization Strategies for Wind-PV Systems in Electricity Markets Assisted by CPS. Energy Procedia 2016, 106, 111-121. [CrossRef]

5. Rana, M.; Xiang, W.; Wang, E.; Li, X.; Choi, B.J. Internet of Things Infrastructure for Wireless Power Transfer Systems. IEEE Access 2018, 6, 19295-19303. [CrossRef]

6. Uchida, A.; Matsui, K.; Shimokawa, S.; Oshima, H. Three-dimensional wireless power transfer method to realize efficient charging of IoT devices. Fujitsu Sci. Tech. J. 2017, 53, 51-56.

7. Li, S.; Mi, C.C. Wireless Power Transfer for Electric Vehicle Applications. IEEE J. Emerg. Sel. Top. Power Electron. 2015, 3, 4-17. [CrossRef]

8. Goh, S.T.; Zekavat, S.R. All electric aircraft mid-air recharging via wireless power transfer: Battery requirement study. In Proceedings of the 6th IEEE International Conference on Wireless for Space and Extreme Environments (WiSEE), Huntsville, AL, USA, 11-13 December 2018; pp. 212-217.

9. Bacelar, T.; Madeiras, J.; Melicio, R.; Cardeira, C.; Oliveira, P.On-board implementation and experimental validation of collaborative transportation of loads with multiple UAVs. Aerosp. Sci. Technol. 2020, 107, 106284. [CrossRef] 
10. Simon, M.J.; Langer, C.; Rubin, S.; Komush, D.; Maciuca, D.B. Wireless power transfer for responsive space applications. In Proceedings of the AIAA Space 2009 Conference \& Exposition, Pasadena, CA, USA, 14-17 September 2009; pp. 1-11.

11. Dghais, W.; Alam, M. Wireless Power Transfer and In-Vehicle Networking Integration for Energy-Efficient Electric Vehicles. Mob. Netw. Appl. 2016, 23, 1151-1164. [CrossRef]

12. Baikova, E.N.; Valtchev, S.S.; Melicio, R.; Pires, V.F.; Krusteva, A.; Gigov, G. Study on electromagnetic emissions from wireless energy transfer. In Proceedings of the IEEE International Power Electronics and Motion Control Conference (PEMC), Varna, Bulgaria, 25-30 September 2016; pp. 492-497.

13. Baikova, E.N.; Valtchev, S.S.; Melicio, R.; Pires, V.F.; Krusteva, A.; Gigov, G. Wireless power transfer impact on data channel. In Proceedings of the IEEE International Symposium on Power Electronics, Electrical Drives, Automation and Motion (SPEEDAM), Amalfi, Italy, 22-24 June 2016; pp. 853-858.

14. Baikova, E.N.; Valtchev, S.; Melício, R.; Pires, V.M. Electromagnetic Interference from a Wireless Power Transfer System: Experimental Results. Renew. Energy Power Qual. J. 2016, 1, 1020-1024. [CrossRef]

15. Baikova, E.N.; Romba, L.; Valtchev, S.; Melício, R.; Pires, V.F.; Krusteva, A.; Gigov, G. Electromagnetic field generated by a wireless energy transfer system: Comparison of simulation to measurement. J. Electromagn. Waves Appl. 2018, 32, 554-571. [CrossRef]

16. Baikova, E.N.; Baikov, A.V.; Valtchev, S.S.; Melicio, R. Frequency Tuning of the resonant wireless energy transfer system. In Proceedings of the IEEE 45th Annual Conference of the Industrial Electronics Society (IECON 2019), Lisbon, Portugal, 14-17 October 2019; pp. 6615-6620.

17. Romba, L.F.; Valtchev, S.S.; Melicio, R. Improving magnetic coupling for battery charging Proceedings. In Proceedings of the IEEE International Power Electronics and Motion Control Conference (PEMC 2016), Varna, Bulgaria, 25-30 September 2016; pp. 291-297.

18. Wang, W.; Wang, H.; Li, Q.; Xu, J.; Meng, T.; Zhang, B.; Zhang, Z. Analysis and Compensation of Incomplete Coupling for Omnidirectional Wireless Power Transfer. Energies 2019, 12, 3277. [CrossRef]

19. Romba, L.F.; Valtchev, S.S.; Melicio, R.; Mudrov, M.V.; Ziuzev, A.M. Electric vehicle battery charger controlled by magnetic core reactor to wireless power transfer system. In Proceedings of the IEEE 17th International Conference on Environment and Electrical Engineering (EEEIC), Milan, Italy, 6-9 June 2017; pp. 1-6.

20. Romba, L.F.; Valtchev, S.S.; Melicio, R. Single-Phase Wireless Power Transfer System Controlled by Magnetic Core Reactors at Transmitter and Receiver; Technological Innovation for Smart Systems; Springer: Cham, Switzerland, 2017; pp. 419-428.

21. Romba, L.F.; Baikova, E.N.; Borges, C.; Melicio, R.; Valtchev, S.S. Wireless Battery Charger for EV with Circular or Planar Coils: Comparison; Technological Innovation for Resilient Systems; Springer: Cham, Switzerland, 2018; pp. 214-223.

22. Romba, L.F.; Valtchev, S.S.; Melicio, R. Three-phase magnetic field system for wireless energy transfer. In Proceedings of the IEEE International Symposium on Power Electronics Electrical Drives, Automation and Motion (SPEEDAM), Capri, Italy, 22-24 June 2016; pp. 73-78.

23. Barmada, S.; Raugi, M.; Tucci, M. Power line communication integrated in a wireless power transfer system: A feasibility study. In Proceedings of the 18th IEEE International Symposium on Power Line Communications and Its Applications, Glasgow, UK, 30 March-2 April 2014; pp. 116-120.

24. Barmada, S.; Dionigi, M.; Mezzanotte, P.; Tucci, M. Design and experimental characterization of a combined WPT-PLC system. Wirel. Power Transf. 2017, 4, 160-170. [CrossRef]

25. Barmada, S.; Raugi, M.; Tucci, M.; Mezzanotte, P.; Dionigi, M. Combining WPT and PLC: A review. In Proceedings of the IEEE 2nd International Forum on Research and Technologies for Society and Industry Leveraging a Better Tomorrow (RTSI), Bologna, Italy, 7-9 September 2017; pp. 1-4.

26. Zouaoui, S.; Souilem, M.; Dghais, W.; Radwan, A.; Barmada, S.; Tucci, M. Wireless power transfer and data communication cognitive radio through two-coil inductive channel. In Proceedings of the IEEE Global Communications Conference (GLOBECOM), Waikoloa, HI, USA, 9-13 December 2019; pp. 1-6.

27. Barmada, S.; Tucci, M. Optimization of a magnetically coupled resonators system for power line communication integration. In Proceedings of the IEEE Wireless Power Transfer Conference (WPTC), Boulder, CO, USA, 13-15 May 2015; pp. 1-4.

28. Barmada, S.; Tucci, M.; Fontana, N.; Dghais, W.; Raugi, M. Design and realization of a multiple accesswireless power transfer system for optimal power line communication data transfer. Energies 2019, 12, 988. [CrossRef] 
29. Houran, M.A.; Yang, X.; Chen, W. Magnetically Coupled Resonance WPT: Review of Compensation Topologies, Resonator Structures with Misalignment, and EMI Diagnostics. Electronics 2018, 7, 296. [CrossRef]

30. Wang, J.; Hu, M.; Cai, C.; Lin, Z.; Li, L.; Fang, Z. Optimization design of wireless charging system for autonomous robots based on magnetic resonance coupling. AIP Adv. 2018, 8, 055004. [CrossRef]

31. Feng, J.; Li, Q.; Lee, F.C. Omnidirectional wireless power transfer for portable devices. In Proceedings of the IEEE Applied Power Electronics Conference and Exposition (APEC), Tampa, FL, USA, 26-30 March 2017; pp. 1675-1681.

32. Yan, Z.; Song, B.; Zhang, Y.; Zhang, K.H.; Mao, Z.; Hu, Y. A Rotation-Free Wireless Power Transfer System with Stable Output Power and Efficiency for Autonomous Underwater Vehicles. IEEE Trans. Power Electron. 2019, 34, 4005-4008. [CrossRef]

33. Zhang, B.; Zhang, B.; Xiao, W.; Qiu, D.; Chen, Y.; Guan, J. Omnidirectional Wireless Power Transfer System Based on Rotary Transmitting Coil for Household Appliances. Energies 2018, 11, 878. [CrossRef]

34. Lin, D.; Zhang, C.; Hui, S.Y.R. Two- and three-dimensional omnidirectional wireless power transfer. IEEE Trans. Power Electron. 2014, 29, 4470-4474. [CrossRef]

35. Lin, D.; Zhang, C.; Hui, S.Y.R. Mathematical analysis of omnidirectional wireless power transfer part-I: Two-dimensional systems. IEEE Trans. Power Electron. 2016, 32, 625-633. [CrossRef]

36. Narayanamoorthi, R.; Juliet, A.V. IoT-enabled home cage with three dimensional resonant wireless power and data transfer scheme for freely moving animal. IEEE Sens. J. 2018, 18, 8154-8161. [CrossRef]

37. Huang, Y.; Zhang, C.; Hui, S.Y.R. Mathematic Analysis of Omnidirectional Wireless Power Transfer-Part-II Three-Dimensional Systems. IEEE Trans. Power Electron. 2016, 32, 613-624. [CrossRef]

38. Feng, J.; Li, Q.; Lee, F.C. Coil and circuit design of omnidirectional wireless power transfer system for portable device application. In Proceedings of the IEEE Energy Conversion Congress and Exposition (ECCE), Portland, OR, USA, 23-27 September 2018; pp. 914-920.

39. He, Z.; Wang, Y.; Ding, L.; Nie, X. Research on three-dimensional omnidirectional wireless power transfer system for subsea operation. In Proceedings of the IEEE Oceans 2017 Aberdeen Conference and Workshop, Aberdeen, UK, 19-22 June 2017; pp. 1-5.

40. Power Line Communication-an Overview (pdf), ScienceDirect Topics. Available online: https://www. sciencedirect.com/topics/engineering/power-line-communication/pdf (accessed on 30 March 2020).

41. Huang, X.; Qiang, H.; Huang, Z.; Sun, Y.; Li, J. The interaction research of smart grid and EV based wireless charging. In Proceedings of the IEEE Vehicle Power and Propulsion Conference (VPPC), Beijing, China, 15-18 October 2013; pp. 354-358.

42. Pinomaa, A.; Ahola, J.; Kosonen, A.; Nuutinen, P. Homeplug green PHY for the LVDC PLC concept: Applicability study. In Proceedings of the IEEE International Symposium on Power Line Communications and Its Applications (ISPLC), Austin, TX, USA, 29 March-1 April 2015; pp. 205-210.

43. Zhang, C.; Huang, Y.; Hui, S.Y. Basic Control Principles of Omni-Directional Wireless Power Transfer. IEEE Trans. Power Electron. 2015, 31, 1. [CrossRef]

44. Aydin, E.; Kosesoy, Y.; Yildiriz, E.; Aydemir, M.T. Comparison of hexagonal and square coils for use in wireless charging of electric vehicle battery. In Proceedings of the IEEE International Symposium on Electronics and Telecommunications (ISETC), Timisoara, Romania, 8-9 November 2018; pp. 1-4.

Publisher's Note: MDPI stays neutral with regard to jurisdictional claims in published maps and institutional affiliations.

(C) 2020 by the authors. Licensee MDPI, Basel, Switzerland. This article is an open access article distributed under the terms and conditions of the Creative Commons Attribution (CC BY) license (http://creativecommons.org/licenses/by/4.0/). 